\title{
Enfermagem do trabalho e o risco de contaminação biológica em tempos de
}

\section{pandemia de Covid-19}

\author{
Occupational nursing and the risks in times of Covid-19 pandemic \\ La enfermería laboral y los riesgos en tiempos de pandemia Covid-19
}

Marcia Bastos Couto

ORCID: https://orcid.org/0000-0003-0114-8691 Universidade Federal Fluminense, Brasil

E-mail: marbcouto@gmail.com

Rose Mary Costa Rosa Andrade Silva

ORCID: https://orcid.org/0000-0002-6403-2349 Universidade Federal Fluminense, Brasil

E-mail:roserosauff@gmail.com

Eliane Ramos Pereira

ORCID: https://orcid.org/0000-0002-6381-3979 Universidade Federal Fluminense, Brasil

E-mail:elianeramos.uff@gmail.com

Lígia D'arc Silva Rocha Prado

ORCID: https://orcid.org/0000-0001-9690-9953

Universidade Federal Fluminense, Brasil

E-mail:enfaligiaprado@hotmail.com

Janaina Mengal Gomes Fabri

ORCID: https://orcid.org/0000-0002-4777-4746

Universidade Federal Fluminense, Brasil

E-mail:janamgfabri@gmail.com

Sandra Conceição Ribeiro Chícharo

ORCID: https://orcid.org/0000-0002-1487-0088

Universidade Federal Fluminense, Brasil E-mail:sandracrchicharo@gmail.com

Carlos Eduardo Peres Sampaio

ORCID: https://orcid.org/0000-0002-6770-7364

Universidade do Estado do Rio de Janeiro, Brasil

E-mail: carlosedusampa @yahoo.com.br

Marcos Andrade Silva

ORCID: https://orcid.org/0000-0001-8960-3171

Fundação de Apoio a Escola Técnica, Brasil E-mail: marcosandrade.uff@gmail.com

\section{Resumo}

Objetivo: refletir acerca da vulnerabilidade dos profissionais de enfermagem diante dos riscos por acidente de trabalho. Conteúdo: trata-se de um estudo teórico-reflexivo, fundamentado em referenciais teóricos sobre acidente no trabalho pensando nos contextos vivenciados por enfermeiros contaminados durante o combate a pandemia da covid19. Considerações finais: Constata-se, com base nesse estudo, que os riscos iminentes no trabalho de enfermagem, refletem nas dimensões individuais e coletiva dos grupos e pacientes sendo uma combinação de elementos e experiências que se entrecruzam devido a emergência causada pela doença provocada pelo novo coronavírus. Destaca-se, as dificuldades encontradas por enfermeiros para além daquelas relacionadas à doença da covid-19, a saber, os riscos de acidentes aumentaram devido a pandemia no Brasil.

Palavras-chave: Enfermagem do trabalho; Infecções por Coronavírus; Vulnerabilidade em saúde pública; Riscos de óbitos.

\section{Abstract}

Objective: Reflectar acerca da vulnerabilidade dos profissionais de enfermagem diante dos riscos por acidente de trabalho. Conteúdo: trata-se de uma análise teórico-reflexiva, fundamentada em referenciais teóricos sobre acidente no trabalho pensando nos contextos vivenciados por enfermeiros e outros profissionais contaminados durante o combate a pandemia da covid-19. Final considerations: Based on this study, it was observed that the imminent risks in the nursing work reflect in the individual and collective dimensions of the groups, professionals and patients, being a combination of elements and experiences that intersect due to the emergency caused by the disease caused by the new 
coronavirus. It stands out, several difficulties encountered by health professionals beyond those related to the covid-19 disease, namely, the risks of accidents increased due to the spread of the disease in full expansion in Brazil.

Keywords: Occupational nursing; Coronavirus infections; Public health vulnerability; Death risks.

\section{Resumen}

Objetivo: reflejar la vulnerabilidad de los profesionales de la enfermería ante los riesgos del ácido laboral. Conteúdo: trata-se de uma análise teórico-reflexiva, fundamentada em referências teóricas sobre o acidente no trabalho pensando nos contextos vividos por enfermeiros e outros profissionais contaminados durante o combate à pandemia do covê-19. Consideraciones finales: Con base en este estudio, se encontró que los riesgos inminentes en el trabajo de enfermería se reflejan en las dimensiones individuales y colectivas de los grupos, profesionales y pacientes siendo una combinación de elementos y experiencias que se cruzan debido a la emergencia causada por la enfermedad provocada por el nuevo coronavirus. Se destaca, varias dificultades encontradas por los profesionales de la salud más allá de los relacionados con la enfermedad de covid-19, a saber, los riesgos de accidentes aumentaron debido a la propagación de la enfermedad en plena expansión en Brasil.

Palabras clave: Enfermería ocupacional; Infecciones por Coronavirus; Vulnerabilidad de la salud publica; Riesgos de muerte.

\section{Introdução}

A enfermagem do trabalho assume papel importante na área da saúde Ocupacional. O Enfermeiro do Trabalho tem competências que lhe permitem realizar atividades visando a promoção da saúde. Além disso, atua na prevenção de acidentes e de doenças relacionadas com o trabalho bem como tem a função de preparar os trabalhadores para situações emergenciais. A prevenção e os cuidados com o trabalhador são atividades que se revestem de grande importância para diminuir o absenteísmo causado pela doença, e para melhorar a produtividade (Oliveira \& Serrano, 2011).

O objetivo deste estudo é refletir sobre os riscos evidenciados na rotina dos profissionais de enfermagem, à frente do combate a pandemia da Covid-19. Um momento difícil e desgastante acirrado pelos riscos causados por acidentes de trabalho. O problema se agravou por saber que o vírus era desconhecido e que cada vez mais os profissionais se tornavam vítimas de contaminação. A pergunta norteadora evidencia os riscos causados por acidente de trabalho bem como apresenta as dificuldades enfrentadas pelos profissionais de enfermagem durante esse período de pandemia da Covid-19. Quais os tipos mais comuns de acidente de trabalho que acontecem durante o combate à pandemia?

Acidente de trabalho se tornou motivo de preocupação no ambiente das grandes empresas, sejam públicas ou privadas. Nessa conjuntura enquadra-se boa quantidade de empregados atuando nas organizações hospitalares e que estão a serviço da população. Os serviços prestados em casos de epidemias ou pandemias, vão desde a convocação em massa de profissionais da saúde para aumentar as chances de combate e evitar o número de mortes, porém aumenta juntamente com os riscos em diversos setores.

De modo que, o volume de atividades aumentou durante a Covid-19, sendo realizadas com mais constância com dobras de turnos, no âmbito da enfermagem ocorreu muitas convocações de profissionais devido à necessidade de combater a doença, evitar as mortes e garantir os cuidados aos contaminados pela Covid-19. O grande número de contaminados gerou extensivo atendimento exigindo dos profissionais, trabalho dobrado.

Em tempos de pandemia, o trabalhador se vê diante de extensa exposição de agentes patogênicos. Embora a recomendação para os profissionais de saúde é que mantenham suas carteiras de vacinação atualizadas no caso da nova variante conhecida como Novo Coronavírus ou Covid-19, o impasse está centrado exatamente na proteção por vacina, inicialmente foi impossível se proteger. O profissional que atende diretamente os pacientes contaminados estava em plena exposição. Vale frisar que foram muitos os acidentes de trabalho ocasionados com profissionais de enfermagem, durante o cumprimento de suas atividades. A doença contaminou uma gama de profissionais da saúde em todas as partes do país. 


\section{Metodologia}

Trata-se, portanto, de um artigo de reflexão sobre o risco de contaminação biológica com foco no profissional de enfermagem.

apresenta-se as análises pelos seguintes descritores: Enfermagem do Trabalho; Infecções por coronavírus; Vulnerabilidade em Saúde pública; Riscos de óbitos por Acidentes. Os resultados apresentam reflexões a partir de relatos de experiência e críticos-reflexivos selecionados sobre a saúde dos profissionais de enfermagem que atuam na linha de frente no atendimento aos pacientes acometidos pela Covid-19.

A pesquisa reflete sobre acidente de trabalho e suas implicações nas rotinas dos profissionais de enfermagem. Enfatizando as possíveis dificuldades e frustrações que afetaram os profissionais de enfermagem durante o enfrentamento da Covid-19. A rotina diária gera exposição aos riscos de acidente como vivenciada por profissionais de saúde. O momento vivenciado nos hospitais denota a necessidade de identificar os impactos causados pela pandemia do Corona Vírus. A saber, a contaminação por acidente de trabalho evidencia bastante a vulnerabilidade e os riscos para os profissionais devido a precariedade dos serviços prestados à saúde pública dos brasileiros.

Desse modo, o estudo contribui para a reflexão sobre contaminação de profissionais de enfermagem por acidentes de trabalho durante o combate à pandemia da Covid-19. Ressalta-se que somatória dos riscos coadunam com outros fatores como esgotamento físico, à exaustão dos plantões. Em vista de toda essa situação o mais agravante é que exige do profissional de saúde o que ele pode oferecer. Ademais, há indícios de que vários profissionais de enfermagem estão sendo acometidos por distúrbios que os privam de executar seu trabalho ou até mesmo afastados por estarem no limite de sua capacidade mental, física e psicológica.

O referencial teórico ancora-se na análise teórica-reflexiva e se fundamenta em análises recorrentes de publicações com intuito de refletir sobre acidente de trabalho por profissionais da saúde, durante o combate a Covid-19. A busca de dados foi realizada em artigos previamente selecionados, identificados e ordenados em revistas eletrônicas. Por conseguinte, os dados extraídos das buscas bibliográficas, buscam responder a três hipóteses consideravelmente relevantes e que levam as seguintes considerações: o medo de ser contaminado e ir a óbito, sucumbe o profissional de enfermagem por ter que lidar com uma doença desconhecida e altamente contagiosa, a Covid-19; as estatísticas trazem reflexões sobre profissionais de enfermagem acometidos pela covid-19 e ressalta-se que os pesquisadores citados apontam para dificuldades encontradas na realidade, tais como a falta de estrutura dos hospitais para o enfrentamento da pandemia Covid-19 e para garantir a segurança dos profissionais preservando-os dos riscos de contaminação. De acordo Silvio de Almeida Junior,2019) A equipe de enfermagem possui um maior risco de aquisição de doenças ocupacionais, devido principalmente ao seu contato direto com fômites contaminantes e elevada frequência de realização de procedimentos que podem ocasionar acidentes.

\section{Discussão}

\section{Acidente de trabalho no Brasil}

O conceito de enfermagem do trabalho, trata-se de mais uma das ramificações das especializações de enfermagem voltadas para saúde pública no Brasil. O profissional de enfermagem do trabalho se utiliza das mesmas técnicas e métodos e se difere apenas por ser um trabalho voltado para prevenção da saúde do trabalhador. A prevenção e proteção contra riscos é importante para evitar acidentes graves durante sua atividade. A enfermagem do trabalho vem assumindo determinada relevância e crescendo como modalidade da área da saúde (Matos et al., 2017). De modo que a enfermagem do trabalho se consolidou como especialidade no final do século XIX na Inglaterra, ampliando os contextos de trabalho profissionalizantes.

No Brasil, a enfermagem do trabalho e demais profissões como segurança e medicina do trabalho foram incorporados nas empresas a partir do início da década de 70. Nesse período, o Brasil se consagrou campeão mundial de acidentes de 
trabalho. Por essa razão, o governo impôs obrigatoriamente que as empresas contratassem profissionais especializados. Embora houvesse a necessidade de diminuir os acidentes no trabalho naquele momento, somente em 2004, o Conselho Federal de Enfermagem - COFEN em sua resolução 290/2004, fixou a especialização de enfermagem de competência do enfermeiro. Sendo assim o profissional passa atuar na enfermagem do trabalho.

O enfermeiro do trabalho vem ocupando espaço dentro das organizações. E atua diretamente envolvido com a qualidade de vida do trabalhador. Com o intuito de prevenir riscos causados por acidente de trabalho (2). Do mesmo modo, o enfermeiro do trabalho pode prestar serviços de assistência de enfermagem aos trabalhadores doentes ou acidentados afastados de suas atividades por acidente. O acompanhamento e o tratamento são fundamentais para o trabalhador para garantir o restabelecimento o bem estar físico e psicológico.

A pandemia Covid-19 é considerada como um desvio de saúde em escalada global, que demanda intervenção emergencial e implica em pensamento crítico pela ausência de preparo e planejamento da assistência no setor da enfermagem. Evidencia-se em várias teorias e fontes jornalísticas, as significativas consequências causadas pela pandemia da Covid-19 no Brasil. Este estudo serve de base para pensar nos desafios e impasses enfrentados pelos profissionais de enfermagem atuantes no Serviço de Emergência.

O conceito adotado pelos profissionais de enfermagem sobre a biossegurança, é consolidado na prevenção e no controle de infecção, numa relação de causa-efeito, e sua prática pode estar ancorada na adequação de condutas e nas técnicas, podendo alterar a forma de cuidar, exigindo, muitas das veze, um distanciamento físico, por parte do profissional, com relação ao cliente, materializando-se em "agir com cautela" (Souza et al., 2016). Todo cuidado é pouco quando se trata da qualificação da equipe de trabalho para reverter quadros de segurança da saúde pública. Reitera-se que os acidentes de trabalho com profissionais de enfermagem geralmente envolvem ferimentos com agulhas ou outro material cortante e contato direto com sangue ou materiais contaminados. Dentre os mais envolvidos com esses acidentes, destacam-se os profissionais de enfermagem.

Geralmente são riscos que podem comprometer tanto a saúde do homem quanto dos animais expandindo para o meio ambiente, atingindo a qualidade de vida dos trabalhadores prejudicando os trabalhos desenvolvidos.

De acordo com os dados fornecidos pelo Ministério da Saúde, pelo menos 484.081 profissionais de saúde foram infectados pela covid-19 durante o exercício da profissão. A saber pelos menos 470 morreram. Reconhecidamente que o número de hábitos chega a 1,3 morte por dia, significa que um profissional morre a cada 19 horas no Brasil (Conselho Federal de Enfermagem [Cofen], 2021). Os dados utilizados nos referenciais metodológicos do Ministério da Saúde mostram como são feitas as classificações de diagnósticos, intervenções e resultados de enfermagem. Esses dados estão ancorados no atendimento de enfermagem tanto para analisar como para consolidar as teorias circundantes sobre riscos de contaminações aos profissionais de saúde por acidente de trabalho durante o combate ao Novo Coronavírus.

Os problemas apresentados vão além dos riscos de contaminação por acidente de trabalho. Surgem outras implicações oriundas da doença que pode levar o profissional a morte (Teixeira et al., 2020). Essa incerteza gera intenso sofrimento psíquico, levando aos transtornos de ansiedade e elevados níveis de estresse levando aos distúrbios do sono. A preocupação do profissional de saúde com acidentes de trabalho não implica em responsabilidade durante a prática incorreta de situação ocasionadas por uma fatalidade com os materiais de proteção, ante implica na vulnerabilidade dos profissionais ocasionados pelas condições precárias de trabalho oferecidas pelo serviço público.

A Lei n 8.213, de 24 de julho de 1991 (Lei n 8.213, 1991) define acidente de trabalho como aquele que ocorre pelo exercício do trabalho a serviço da empresa, pelo exercício do trabalho provocando lesão corporal ou perturbação funcional que cause a morte, a perda ou a redução permanente ou temporária da capacidade para o trabalho.

Também são considerados acidentes de trabalho, segundo Mallmann, (2016) aqueles ocorridos durante o percurso 
que o trabalhador faz da sua casa para o trabalho ou o contrário.

A saúde do trabalhador se faz presente no contexto interdisciplinar, priorizando sua atuação, identificando os prováveis riscos, visando melhorar este ambiente. (Alcantara, 2019)

Sob a perspectiva da prática profissional "o maior empreendimento do enfermeiro do trabalho está em contribuir para evitar os acidentes e doenças, pela identificação e eliminação dos riscos existentes no ambiente de trabalho" (Lima \& Lima, 2017, p.1). No contexto brasileiro os riscos agravam-se devido a precariedade dos serviços públicos oferecidos e tudo pode piorar quando se está diante de uma crise pandêmica. No âmbito da enfermagem do trabalho surge a necessidade de preservação da saúde física, por meio de equipamentos cientificamente recomendados para diminuir os riscos de contaminação.

A condição insatisfatória do trabalho tem como consequência a perda da capacidade laboral do trabalho em sua totalidade, assim como pode ocasionar doenças ocupacionais ou acidentes de trabalho que levam ao afastamento temporário ou permanente do trabalho e de suas atividades habituais. Levar este saber ao trabalhador deve fazer parte das medidas de prevenção. Nesta questão, portanto, segundo Silvia, 2016 cabe ao enfermeiro ser conhecedor do processo de gerenciamento para influenciar a sua equipe.

Em alguns casos, as medidas a serem tomadas são mais emergenciais. Geralmente quando acidentes acontecem com materiais contaminados, o mais recomendável é a limpeza e higienização do local lesionado imediatamente (Baptista et al., 2018). Em seguida se notificar a chefia para tomada de providência imediata. Embora acidentes de trabalho (AT) ocorrem no mundo todo, tanto em países desenvolvidos como naqueles em subdesenvolvidos. Todavia, a preocupação com o trabalhador se dá pelo afastamento do trabalho em idade produtiva. Além do de danos físicos e psicológicos causados em caso de acidentes que podem levar à morte (Gallasch et al., 2020).

De todo modo, é preciso buscar compreender a vulnerabilidade desses profissionais tendo em vista os efeitos dos acidentes no exercício profissional. Aumenta o número de profissionais que reclamam de adoecimento devido à sobrecarga de trabalho por dobra de turno. A quantidade de enfermeiros que reivindicam seus direitos durante a pandemia da Covid-19 demonstra os constates desafios. "E como dificilmente poder-se-á mudar a natureza do trabalho da enfermagem, apontada como preditora do adoecimento, é inegável a importância de se reduzir a carga horária destes profissionais" (Pontes et al., 2018, p. 4).

Os acidentes de trabalho podem ocorrer ainda porque alguns trabalhadores ignoram as normas exigidas deixando de cumprir os protocolos de atendimento. Entre os fatores de riscos causados pela Covid-19, vem se destacando risco biológico no ambiente hospitalar. Esse fator de risco está ligado diretamente aos profissionais da frente de combate e controle das contaminações devido "à permanente exposição dos trabalhadores de enfermagem aos agentes biológicos existentes no ambiente hospitalar" (Joaquim et al., 2020, p. 5). Os riscos implicam em ser ferido no momento do acidente de trabalho associam-se aos desafios enfrentados durante os atendimentos aos pacientes contaminados.

As limitações encontradas para realização deste estudo, restringiram-se aos embasamentos empíricos não coletados, dado ao momento vivenciado nos hospitais pelos profissionais de enfermagem. Apesar da diminuição dos casos de contaminados ainda não é possível contabilizar e compreender a expansão dos casos de acidentes de trabalho. Primeiro, devido a extensão da pandemia na população brasileira sabendo que a doença é um fenômeno presente na sociedade.

Neste sentido, primeiro está "o cuidado de evitar a contaminação dos trabalhadores nesta pandemia pelo novo coronavírus deve ser priorizado" (Gallasch et al., 2020, p. 1). Segundo, pelos riscos ocasionados para o pesquisador que deseja investigar o campo prático. E terceiro, a sensibilidade dos profissionais que forma contaminados em seu ambiente de trabalho, porém não sente à vontade para falar de suas vivências.

As contribuições deste estudo, evidenciam as dificuldades, apresenta os desafios para enfermeiros do trabalho bem 
como mostra a necessidade de compreensão do problema, pelo olhar de outros profissionais atuando nos diversos campos da saúde pública. Como se definir o foco desses profissionais que tem sido exatamente o controle e o combate da pandemia da Covid-19, e com isso, evitar a contaminação de um número maior de profissionais de enfermagem, por acidente de trabalho. Acidente de trabalho é um assunto a ser investigado e debatido a luz de outros questionamentos tais como prevenção e diminuição dos riscos de contaminações dos profissionais. A urgência incide em controlar ao máximo a doença. Embora é evidente a vulnerabilidade dos profissionais de enfermagem do trabalho em tempos de pandemia da Covid-19.

\section{Considerações}

O campo da enfermagem do trabalho na atualidade encontra-se diante de um grande desafio para além do combate à pandemia da Covid-19. A realidade apresenta a necessidade de debater propostas voltadas para a preservação da saúde, seja física ou psicológica. Os profissionais que mais se destacaram durante os atendimentos ao coronavírus estavam atuando diretamente na frente ou na tentativa de controle da doença. Todos os trabalhos envolvendo profissionais de saúde são realizados para amenizar os estragos ocasionados. Reconhece-se a importância de ampla divulgação sobre os efeitos da pandemia considerando seus impactos para os profissionais de saúde, em todos os meios de comunicação.

A prevenção de acidente de trabalho com material biológico é uma importante etapa na prevenção da contaminação de trabalhadores da saúde por patógenos de transmissão sanguínea. Dados epidemiológicos sobre os acidentes, incluindo as circunstâncias associadas com a transmissão ocupacional por estes patógenos, são essenciais para o direcionamento e a avaliação das intervenções nos níveis local, regional e nacional. De todo modo, a enfermagem do trabalho, como especialidade, busca aprofundar, desenvolver conhecimentos e ampliar seu papel junto à área de saúde do trabalhador. Porém, os acidentes de trabalho com exposição a material biológico é uma realidade preocupante nas instituições de saúde.

Por fim, no escopo deste estudo fica evidenciado que os profissionais de saúde serão os últimos a se recuperar dos danos causados pela pandemia. Restando-lhes traumas, angústias e crises geradas pela sensação de impotência em face da vida e da morte. O esforço é frequente não somente para cuidar desse quantitativo exagerado de pacientes, dentre outras questões, traz-se a lume os riscos por acidente de trabalho evitando conjuntamente os riscos de morte consequentemente para os profissionais da saúde no combate a pandemia da Covid-19.

\section{Referências}

Baptista, A. T. P., Souza, N. V. D. de O., Gallasch, C. H., Varella, T. C. M. Y. M. L., Noronha, I. D. R., \& Noronha, I. D. R. (2018). Adoecimento de trabalhadores de enfermagem no contexto hospitalar. Revista Enfermagem UERJ, 26, e31170. https://doi.org/10.12957/reuerj.2018.31170

Borges, A., De Castro, S., Teixeira Costa De Sousa, J., Amaro, A., \& Santos, D. (2010). Nursing: attributions of the nurse of the work in the prevention of occupational risks. J Health Sci Inst, 28(1), 5-7. https://repositorio.unip.br/wp-content/uploads/2020/12/V28_n1_2010_p5-7.pdf

Conselho Federal de Enfermagem - Cofen (B). Representa um terço das mortes de profissionais de Enfermagem por Covid-19. (2021). http://www.cofen.gov.br/brasil-responde-por-um-terco-das-mortes-de-profissionais-de-enfermagem-por-covid-19_84357.html

Gallasch, C. H., Cunha, M. L. da, Pereira, L. A. de S., \& Silva-Junior, J. S. (2020). Prevention related to the occupational exposure of health professionals workers in the COVID-19 scenario. Revista Enfermagem UERJ, 28, e49596. https://doi.org/10.12957/reuerj.2020.49596

Joaquim, F. L., Silva, R. M. C. R. A., Pereira, E. R., Camacho, A. C. L. F., \& Melo, S. H. S. (2020). Percebendo o outro: Relato de experiência sobre a entrevista fenomenológica. Research, Society and Development, 9(5), e55953175. https://doi.org/10.33448/rsd-v9i5.3175

Lei n 8.213, de 24 de julho de 1991. (1991). Dispõe sobre os Planos de Benefícios da Previdência Social e dá outras providências., nº 8.213 (1991). http://www.planalto.gov.br/ccivil_03/leis/18213cons.htm

Lino, M. M., Nora, P. T., Lino, M. M., \& Furtado, M. (2012). Enfermagem do Trabalho à Luz da Visão Interdisciplinar. Saúde \& Transformação Social, 3(1), 85-91. http://pepsic.bvsalud.org/scielo.php?script=sci_arttext\&pid=S2178-70852012000100014\&lng=pt\&nrm=isoPenna

Magagnini, M. A. M., Rocha, S. A., \& Ayres, J. A. (2011). O significado do acidente de trabalho com material biológico para os profissionais de enfermagem. Revista Gaúcha de Enfermagem, 32(2), 302-308. https://doi.org/10.1590/S1983-14472011000200013

Matos, D. A. R., Silva, S. O. P., \& Lima, C. B. (2017). Nursing of work: addressing competencies and skills for the nurses 'performance. 17(3). https://temasemsaude.com/wp-content/uploads/2017/10/17314.pdf 
Research, Society and Development, v. 10, n. 16, e515101623814, 2021

(CC BY 4.0) | ISSN 2525-3409 | DOI: http://dx.doi.org/10.33448/rsd-v10i16.23814

Oliveira, António José Eugénio de, \& Serrano, M. (2011). Enfermagem em Saúde Ocupacional. Millenium, 115-122. https://doi.org/http://hdl.handle.net/10400.19/1230

Penna, P. M. M., Aquino, C. F., Castanheira, D. D., Brandi, I. V., Cangussu, A. S. R., Macedo Sobrinho, E., Sari, R. S., Silva, M. P. da, \& Miguel, Â. S. M. (2010). Biossegurança: Uma revisão. Arquivos do Instituto Biológico, 77(3), 555-565. https://doi.org/10.1590/1808-1657v77p5552010 Teixeira P.,

Valle S. (1996). Biossegurança: uma abordagem multidisciplinar. Fiocruz.

Pontes, A. P. M. de, Oliveira, D. C. de, Nogueira, V. P. F., \& Machado, Y. Y. (2018). Representações Sociais da Biossegurança no Contexto do HIV/Aids: Contribuições para Saúde do Trabalhador. Revista Enfermagem UERJ, 26, e21211. https://doi.org/10.12957/reuerj.2018.21211

Silva, S. L. da. (2005). Interações do enfermeiro do trabalho com a saúde do trabalhador em âmbito de prática e assistência de enfermagem [Tese de Doutorado, Universidade Federal do Rio de Janeiro. Escola de Enfermagem Anna Nery].

Teixeira, C. F. de S., Soares, C. M., Souza, E. A., Lisboa, E. S., Pinto, I. C. de M., Andrade, L. R. de, \& Espiridião, M. A. (2020). The health of healthcare professionals coping with the Covid-19 pandemic. Ciência \& Saúde Coletiva, 25(9), 3465-3474. https://doi.org/10.1590/1413-81232020259.19562020 